\title{
Agricultural area increases the infection risk of free ranging birds to St. Louis encephalitis and West Nile viruses (Flavivirus)
}

\author{
Ana Mansilla ${ }^{1}$, Juan Grande ${ }^{1}$, and Adrián Diaz ${ }^{2}$ \\ ${ }^{1}$ Colaboratorio de Biodiversidad, Ecología y Conservación (ColBEC), FCEyN-UNLPam, \\ Avenida Uruguay 151 Santa Rosa, La Pampa, AR 6300 \\ ${ }^{2}$ Laboratorio de Arbovirus - Instituto de Virología "Dr. J. M. Vanella" - Facultad de \\ Ciencias Médicas - Universidad Nacional de Córdoba. Ciudad Universitaria, Enfermera \\ Gordillo Gómez s/n, CP X5016GCA Córdoba, Argentina.
}

March 31, 2021

\begin{abstract}
Anthropogenic changes affect biological communities of host and vectors driving arbovirus activity. In general, urban and agricultural ecosystems harbor less avian and mosquito diversity than native ecosystems and are dominated by few species. Human activities have led to the emergence / re-emergence of different infectious pathogens particularly arboviruses representing a threat to both public health and biodiversity. Saint Louis encephalitis (SLEV) and West Nile viruses (WNV) are transmitted by Culex spp. mosquitoes as main vectors and several bird species as hosts. With the aim to study the exposure of free-ranging bird communities to SLEV and WNV in Pampean agroecosystems as well as to evaluate the environmental/ biological factors potentially associated we collected and bleed free ranging birds in 12 sites. Serum samples were analyzed by Plaque Reduction Neutralization Test (PRNT) for both viruses. Generalized Linear Mixed Models (GLMM) were performed to analyze the association between environmental / biological variables from each sampled site and avian exposition. A total of 1019 birds were sampled during 2017 - 2019. Overall, we found SLEV NTAb in 60 out of 1019 samples (5.8\%) while WNV seroprevalence was $2.1 \%$ (21/1019). SLEV and WNV seroprevalence were different among the sampled sites. Agricultural area was positively associated with the SLEV-WNV infection risk for an avian host. Forested area also had a strong association but in a negative way. Our results suggest that open agricultural area increase the infection risk of free ranging birds to SLEV and WNV while forested area diminishes the infection risk maybe through a dilution effect of vector and host communities.
\end{abstract}

\section{Hosted file}

Main text file.pdf available at https://authorea.com/users/405180/articles/516192agricultural-area-increases-the-infection-risk-of-free-ranging-birds-to-st-louisencephalitis-and-west-nile-viruses-flavivirus 

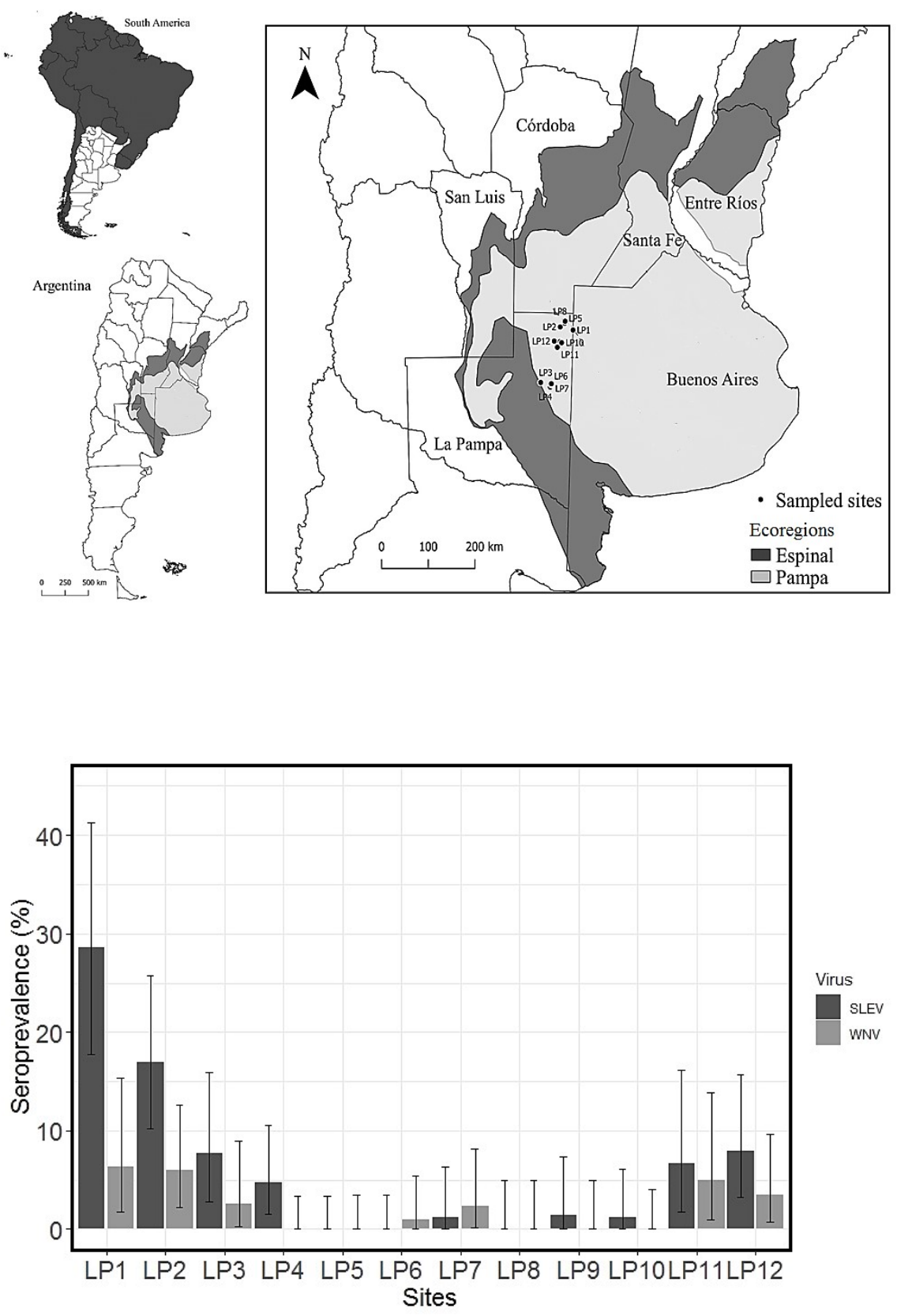